\title{
L-Arginine Utilization by Pseudomonas Species
}

\author{
By VICTOR STALON* AND ANNICK MERCENIER+ \\ Laboratoire de Microbiologie, Faculté des Sciences, Université Libre de Bruxelles, \\ 1 Avenue Emile Gryson, B-1070 Brussels, Belgium
}

(Received 6 July 1983; revised 16 August 1983)

\begin{abstract}
The utilization of arginine was studied in several different Pseudomonas species. The arginine decarboxylase and agmatine deiminase pathways were found to be characteristic of Pseudomonas species of group I as defined by Palleroni et al. (1974). Pseudomonas putida strains had three distinct arginine catabolic pathways initiated by arginine decarboxylase, arginine deiminase and arginine oxidase, respectively. The two former routes were also present in $P$. fluorescens and $P$. mendocina and in $P$. aeruginosa which also used arginine by a further unknown pathway. None of these pathways occurred in P. cepacia strains; agmatine catabolism seemed to follow an unusual route involving guanidinobutyrate as intermediate.
\end{abstract}

\section{INTRODUCTION}

Several different pathways for arginine utilization by micro-organisms have been described (Abdelal, 1979) and are outlined in Fig. 1. These will be referred to as (i) the arginine oxidase pathway, (ii) the arginine decarboxylase pathway, (iii) the arginine deiminase pathway and (iv) the arginase pathway. In some bacteria, more than one pathway for arginine utilization has been observed. For example, Bacillus licheniformis and the cyanobacterium Aphanocapsa use the arginase and the arginine deiminase routes (Broman et al., 1978; Weathers et al., 1978). Arginine can be deiminated and decarboxylated by Pseudomonas aeruginosa (Mercenier et al., $1980 a, b$ ) and by Aeromonas formicans (Stalon et al., 1982). In Klebsiella aerogenes, the arginine decarboxylase/agmatinase route and an unknown arginine amidinotransferase pathway have been reported (Friedrich and Magasanik, 1978; 1979).

In our study of arginine utilization by prokaryotes, we investigated the occurrence of these catabolic pathways in various species of Pseudomonas. Growth of the strains on potential intermediates of arginine metabolism was assessed either by the analysis of the products which accumulated in the culture medium or by measurement of enzyme activities.

\section{METHODS}

Chemicals. Arginine, ornithine, citrulline, putrescine and guanidinobutyrate, and L-amino acid oxidase from Crotalus adamenteus, were obtained from Sigma. $\mathrm{DL}\left[1^{-1+} \mathrm{C}\right]$ Arginine. $\mathrm{HCl}$ was purchased from CEA-IRE, Fleurus (Belgium). Agmatine was obtained from Aldrich and found to be free from putrescine. $N$-Carbamoylputrescine and $N$-[carbamoyl ${ }^{14} \mathrm{C}$ ]carbamoylputrescine were prepared as previously described (Mercenier $e t$ al., 1980a). 2 Ketoarginine was synthesized according to Meister $(1952,1953)$. Arginine was oxidized enzymically by L-amino acid oxidase for $12 \mathrm{~h}$, after which the solution was filtered using a Diaflo apparatus, the enzyme recovered, and the filtrate was concentrated under reduced pressure, 2-Ketoarginine was crystallized at $4{ }^{\circ} \mathrm{C}$ and the precipitate redissolved in hot water. Residual arginine was separated from 2-ketoarginine by chromatography on Dowex $50 \mathrm{WX8}\left(\mathrm{H}^{+}\right.$form $)$. The column $(2.5 \times 30 \mathrm{~cm})$ was washed with 11 water, which eluted the 2-ketoarginine, the arginine remaining adsorbed. The eluate was concentrated and 2 -ketoarginine was recrystallized at $4{ }^{\circ} \mathrm{C}$. A $5 \%$ $(w / v)$ solution was found essentially free of arginine as determined by amino acid analysis (Mercenier et al.,

† Present address: Transgène, S.A., Rue Humann 11, F-67000 Strasbourg, France. 

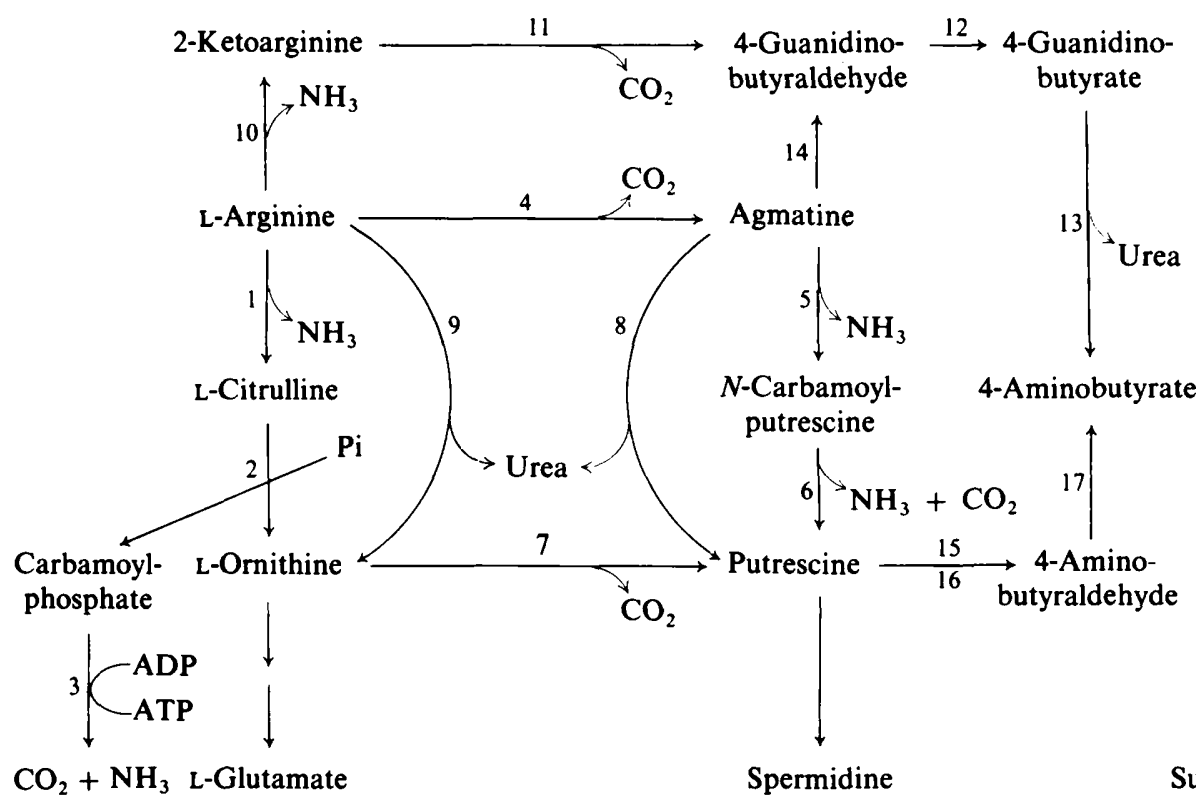

$N$-Carbamoylputrescine

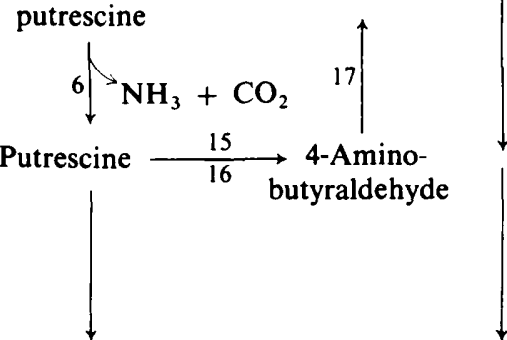

Fig. 1. Pathways of arginine catabolism. The enzymes involved are denoted by the numbers beside the arrows : 1, arginine deiminase (EC 3.5.3.6); , catabolic ornithine carbamoyltransferase (EC 2.1.3.3); 3, carbamate kinase (EC 2.7.2.2); 4, arginine decarboxylase (EC 4.1.1.19); 5, agmatine deiminase (EC 3.5.3.12); 6, $\mathrm{N}$-carbamoylputrescine hydrolase (EC 3.5.3.-); 7, ornithine decarboxylase (EC 4.1.1.17); 8, agmatinase (EC 3.5.3.11); 9, arginase (EC 3.5.3.1); 10, arginine oxidase (EC 1.4.3.-); 11, 2-ketoarginine decarboxylase (EC 4.1.1.-); 12, 4-guanidinobutyraldehyde oxidoreductase (EC 1.2.1.-); 13, guanidinobutyrase (EC 3.5.3.7); 14, agmatine oxidase (EC 1.4.3.-); 15, putrescine transaminase (EC 2.6.1.-); 16, putrescine oxidase (EC 1.4.3.10); 17, aminobutyraldehyde dehydrogenase (EC 1.2.1.19).

1980a). 2-Ketoarginine visualized as a guanidino or 2-keto derivative migrated as a single spot on descending paper chromatography according to Miller \& Rodwell (1971).

Bacterial strains. The strains and their origins are listed in Table 1 . These strains were routinely grown at $30^{\circ} \mathrm{C}$, on nitrogen-free minimal 154 (Stalon et al., 1967). Substrates used as sources of carbon or nitrogen were added after separate sterilization. Unless otherwise stated, the final concentration of metabolites was $10 \mathrm{~mm}$. Rich medium was mineral medium 154 supplemented with yeast extract $0.4 \%(w / v)$.

Growth of the bacteria. In order to screen bacteria able to assimilate potential intermediates of arginine catabolism, each strain was cultivated at $30^{\circ} \mathrm{C}$ with aeration for 1 to $3 \mathrm{~d}$ in $5 \mathrm{ml}$ liquid medium supplemented with the substrate. Bacterial growth was followed by measuring the optical density at $660 \mathrm{~nm}$ with a Beckman photometer. For enzyme activity determinations the cells were grown in $100 \mathrm{ml}$ medium in 11 flasks with vigorous shaking. The cells were collected after $12 \mathrm{~h}$ of growth. The method used for cell growth under limited oxygen tension was carried out as previously described (Mercenier et al., 1980 b).

Enzyme assays. All enzymes were assayed at $37^{\circ} \mathrm{C}$. Catabolic ornithine carbamoyltransferase of Pseudomonas was determined as previously described (Stalon et al., 1972), except that the buffer was adjusted to $\mathrm{pH} 7.5$. Catabolic ornithine carbamoyltransferase of Aeromonas was determined according to Stalon $e t$ al. (1982). The Pseudomonas arginine decarboxylase assay of Mercenier et al. (1980a) was modified by using $0.5 \mathrm{~mm}$-arginine. The Aeromonas arginine decarboxylase determination was according to Stalon et al. (1982). Arginine deiminase was determined according to Stalon et al. (1972), agmatine deiminase following Mercenier et al. (1980a), guanidinobutyrase according to Chou \& Rodwell (1972), and agmatinase by the method of Friedrich \& Magasanik (1979). Qualitative in vitro assays for $N$-carbamoylputrescine hydrolase were carried out by adapting the method described by Tabor et al. (1976) for the detection of ${ }^{14} \mathrm{CO}_{2}$. Single clones were grown at $30^{\circ} \mathrm{C}$ in separate wells of microtitre plates; each well contained $100 \mu \mathrm{l}$ rich medium with $50 \mu \mathrm{M}$-[ureido- $\left.{ }^{-14} \mathrm{C}\right]$ carbamoylputrescine $\left(10^{6}\right.$ c.p.m. $\left.\mu \mathrm{mol}^{-1}\right)$. A Ba(OH $)_{2}$ impregnated Whatman paper no. 3 was placed over each plate. After $24 \mathrm{~h}$, the paper was removed, dried and autoradiographed to assess the production of ${ }^{14} \mathrm{CO}_{2}$ by each culture. 
The protein of the culture extracts was determined by the Lowry method. Specific activity is defined as the amount of enzyme catalysing the formation of $1 \mu \mathrm{mol}$ product $\mathrm{h}^{-1}$ (mg protein) ${ }^{-1}$.

Analytical methods. The presence of intermediary products of arginine catabolism was assayed in the culture supernatants by means of an amino acid analyser (Mercenier et al., 1980a). Citrulline, $N$-carbamoylputrescine and urea were also detected by the colorimetric method of Archibald (1944).

\section{RESULTS AND DISCUSSION}

The aerobic pseudomonads have been divided, on the basis of RNA homology, into five groups (Palleroni et al., 1974), four of which contain the species tested in our study (Table 1). Group $I$ is subdivided into three DNA homology groups, one subgroup corresponds to $P$. fluorescens and $P$. putida, another to P. aeruginosa, P. stutzeri, P. mendocina, P. pseudoalcaligenes, and the third to $P$. syringae. Pseudomonas cepacia is in group II. Pseudomonas acidovorans and $P$. testosteroni have been assigned to group III and group V contains $P$. maltophila.

The nutritional characteristics which we observed for the Pseudomonas strains (Table 1) were consistent with the data of Stanier et al. (1966) with only minor discrepancies. For example, $P$. pseudoalcaligenes was found to use ornithine only as a nitrogen source although it had been reported to grow on this metabolite as a carbon source. With respect to substrates utilization, variations appeared between species of the same subgroup (Table 1) and also between strains of the same species. The two $P$. cepacia strains displayed different phenotypes: one used citrulline and $\mathrm{N}$-carbamoylputrescine as carbon sources, whereas the other used them only as nitrogen sources. Utilization of citrulline as a carbon source appeared to be restricted to relatively few strains. It has been suggested that the rate of citrulline uptake in $P$. aeruginosa may be the ratelimiting step for its utilization (Rahman et al., 1980). The utilization of arginine as a carbon source was restricted to some of the species assigned to group I and to $P$. cepacia (Table 1 ). Among the other group I species, $P$. stutzeri, $P$. pseudoalcaligenes and $P$. syringae used arginine only as a nitrogen source. The group III species, $P$. testosteroni and $P$. acidovorans, did not grow on arginine and utilized only a few of the potential arginine catabolites and thus appear less versatile than the other Pseudomonas species. It should be noted that the P. acidovorans strains are now classified as Xanthomonas (J. De Ley, personal communication).

\section{The arginine oxidase pathway and 2-ketoarginine utilization}

Pseudomonas putida $\mathrm{P}_{2}$ ATCC 25571 catabolizes arginine by oxidative deamination leading to 2-ketoarginine; this step is followed by a decarboxylation giving guanidinobutyraldehyde, which is then converted to guanidinobutyrate (Fig. 1, enzymes 10,11,12) (Miller \& Rodwell, 1971; Chou \& Rodwell, 1972; Vanderbilt et al., 1975). Pseudomonas strains that utilized 2ketoarginine and guanidinobutyrate were characterized by an abundant release of urea into the culture medium. They exhibited a guanidinobutyrase activity (enzyme 13) induced during growth of the cells on these two substrates (Table 2). All the $P$. putida strains excreted urea and also had a high level of guanidinobutyrase when grown on arginine. The induction of arginase activity in Bacillus licheniformis during growth on arginine leads to the production of urea (Broman et al., 1978). Escherichia coli also produces urea when grown on arginine as nitrogen source and this results from the formation of agmatine which is cleaved into putrescine and urea by an agmatinase (enzyme 8) (Morris \& Pardee, 1971). Thus, only $P$. putida appears to use arginine oxidase as the first step for the catabolism of arginine. Although $P$. stutzeri ATCC $17588, P$. testosteroni and $A$. formicans could use 2-ketoarginine as nitrogen source, no guanidinobutyrase was detected either in crude extracts or in toluenized cells.

\section{The arginine decarboxylase activity}

Arginine decarboxylase (enzyme 4) activity has been observed in cell-free extracts of $E$. coli (Morris \& Pardee, 1971), P. aeruginosa (Mercenier et al., 1980a) and Aeromonas formicans (Stalon et al., 1982), grown in the presence of arginine. All the Pseudomonas species of group I exhibited arginine decarboxylase activity (Table 3 ). The enzyme was stimulated by both $0.5 \mathrm{mM}-$ 


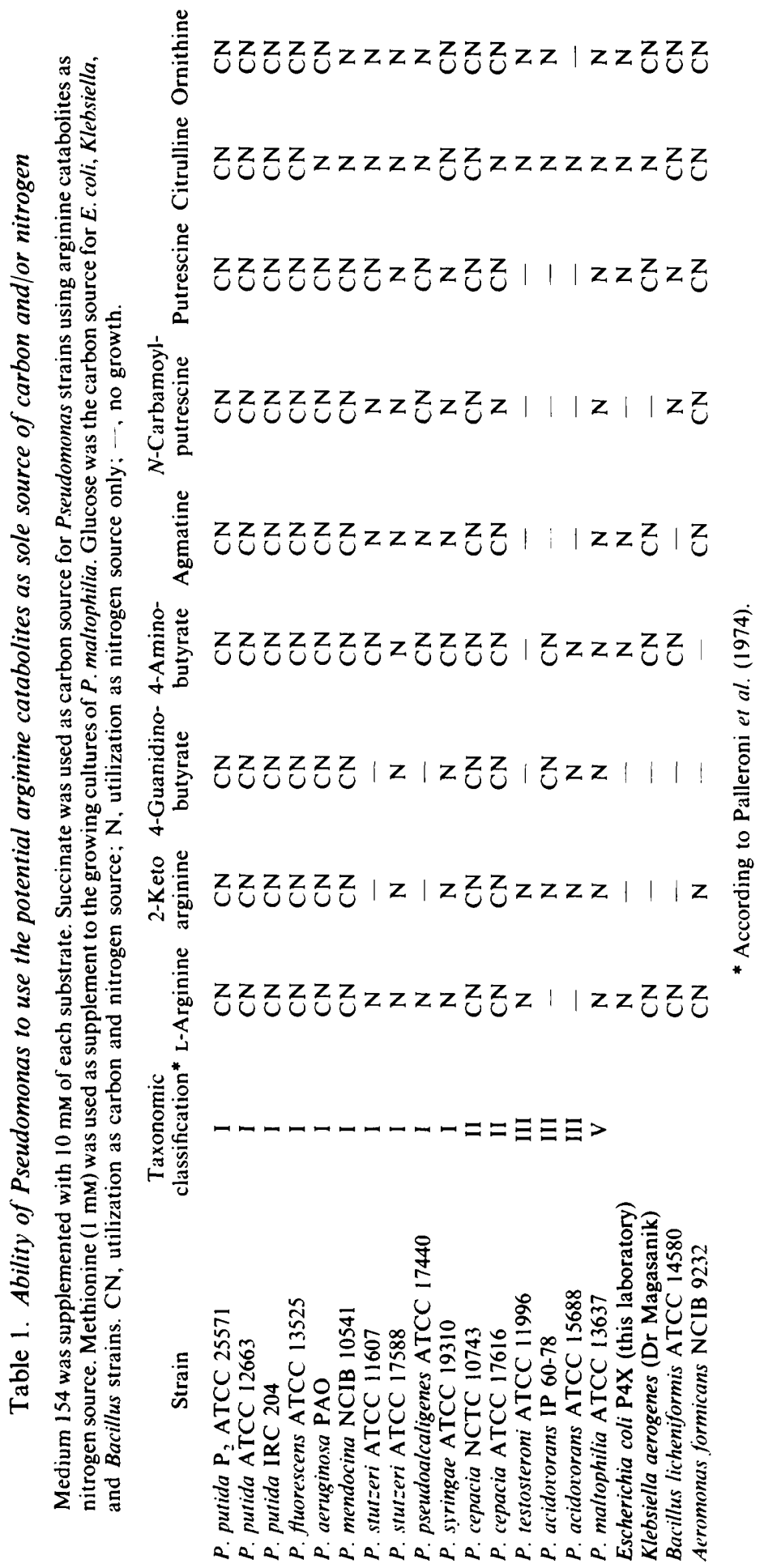


Table 2. Guanidinobutyrase activity in Pseudomonas species grown on different media

Bacteria were grown on medium 154 with $10 \mathrm{~mm}$ of the different substrates as sole source of carbon and nitrogen. Enzyme was assayed as described in Methods. Specific activities are recorded as $\mu \mathrm{mol}$ product formed $(\mathrm{mg} \text { protein })^{-1} \mathrm{~h}^{-1}$.

\begin{tabular}{|c|c|c|c|c|}
\hline \multirow[b]{2}{*}{ Strain } & \multicolumn{4}{|c|}{ Specific activity of guanidinobutyrase after growth on: } \\
\hline & $\begin{array}{l}\text { Minimal } \\
\text { medium* }\end{array}$ & Arginine & 2-Ketoarginine & Guanidinobutyrate \\
\hline P. aeruginosa PAO & 0.4 & $0 \cdot 9$ & 110 & 110 \\
\hline P. putida ATCC 12633 & $<0.2$ & 40 & 63 & 63 \\
\hline P. putida ATCC 25571 & $<0.2$ & 32 & 45 & 74 \\
\hline P. putida IRC 204 & $<0 \cdot 2$ & 34 & 70 & 50 \\
\hline P. mendocina NCIB 10541 & $<0.2$ & $2 \cdot 6$ & 60 & 50 \\
\hline P. fluorescens ATCC 13525 & $<0 \cdot 2$ & 1 & 129 & 150 \\
\hline P. cepacia NCTC 10743 & $<0 \cdot 2$ & $0 \cdot 5$ & 14 & 60 \\
\hline P. cepacia АТCC 17616 & $<0.2$ & $0 \cdot 2$ & 60 & 50 \\
\hline P. acidovorans IP 60-78 & $<0 \cdot 2$ & NT & $58 \dagger$ & $56 \dagger$ \\
\hline P. syringae ATCC 19310 & $<0 \cdot 2$ & $0.2 \dagger$ & NT & $3 \dagger$ \\
\hline
\end{tabular}

\section{Table 3. Formation of arginine decarboxylase and agmatine deiminase in Pseudomonas}

Enzymes were assayed as described in Methods. Specific activities are recorded as $\mu$ mol product formed (mg protein $)^{-1} \mathrm{~h}^{-1}$.

\begin{tabular}{|c|c|c|c|c|c|c|}
\hline \multirow[b]{2}{*}{ Strain } & \multirow{2}{*}{$\begin{array}{l}\text { Growth } \\
\text { medium }\end{array}$} & \multicolumn{2}{|c|}{$\begin{array}{l}\text { Specific activity of } \\
\text { arginine decarboxylase }\end{array}$} & \multicolumn{3}{|c|}{$\begin{array}{l}\text { Specific activity of } \\
\text { agmatine deiminase }\end{array}$} \\
\hline & & Minimal* & Arginine $\dagger$ & Minimal* & Arginine $\dagger$ & Agmatine $\dagger$ \\
\hline P. putida ATCC 25571 & & $0 \cdot 2$ & $0 \cdot 8$ & $0 \cdot 2$ & $0 \cdot 6$ & $2 \cdot 2$ \\
\hline P. putida ATCC 12633 & & 0.02 & $0 \cdot 6$ & $0 \cdot 1$ & 0.7 & $5 \cdot 2$ \\
\hline$P$. putida IRC 204 & & 0.02 & $0 \cdot 9$ & $0 \cdot 2$ & $0 \cdot 6$ & $4 \cdot 0$ \\
\hline P. fluorescens ATCC 13525 & & 0.05 & 0.5 & $0 \cdot 4$ & 0.7 & $2 \cdot 5$ \\
\hline$P$. aeruginosa $\mathrm{PAO}$ & & $0 \cdot 02$ & 1.9 & $0 \cdot 8$ & $1 \cdot 0$ & $30 \cdot 0$ \\
\hline P. mendocina NCIB 10541 & & $0 \cdot 2$ & $0 \cdot 6$ & 0.4 & 4.4 & $25 \cdot 0$ \\
\hline P. pseudoalcaligenes ATCC & 17440 & $0 \cdot 3$ & $0 \cdot 8 \ddagger$ & $0 \cdot 3$ & 1.6 & $4.0 \ddagger$ \\
\hline$P$. stutzeri ATCC 11607 & & 0.5 & $0.5^{+}$ & $0 \cdot 3$ & $2 \cdot 0$ & $9 \cdot 2 t$ \\
\hline$P$. slringae ATCC 19310 & & 0.06 & $0.05^{+}$ & 0.4 & 0.8 & $4 \cdot 6_{+}^{+}$ \\
\hline Aeromonas formicans NCIB & 9232 & 0.05 & $0 \cdot 3$ & 0.5 & $0 \cdot 4$ & $6 \cdot 0^{\circ}$ \\
\hline Escherichia coli $\mathbf{P} 4 \mathrm{X}$ & & NT & $0.6 \S$ & NT & NT & NT \\
\hline
\end{tabular}

* Minimal medium was mineral salt medium 154 supplemented with 10 mM-succinate and 5 mM-( $\left.\mathrm{NH}_{4}\right)_{2} \mathrm{SO}_{4}$ for $P$ seudomonas strains: glucose was used instead of succinate for $E$. coli.

+ Arginine and agmatine media were medium 154 supplemented $10 \mathrm{~mm}$-arginine or $10 \mathrm{~mm}$-agmatine, respectively.

$\ddagger$ Cultures were grown in the presence of $10 \mathrm{~mm}$-succinate.

$\S$ Cultures were grown in the presence of $10 \mathrm{~mm}$-glucose

pyridoxal phosphate and magnesium ions, but thiamin pyrophosphate, the co-factor of the 2ketoarginine decarboxylase (enzyme 11), was without effect.

Pseudomonas stutzeri ATCC 11607 had constitutive arginine decarboxylase activity but with the other species the synthesis of arginine decarboxylase was induced by growth in the presence of arginine (Table 3). Pseudomonas testosteroni and $P$. cepacia had no arginine decarboxylase, although $P$. cepacia could grow on the enzyme product, agmatine.

\section{The agmatine deiminase pathway}

Since agmatine was utilized for growth by most of the Pseudomonas strains of group I, we investigated whether they excreted urea or $N$-carbamoylputrescine. Agmatine utilization by $E$. 
Table 4. Regulation of catabolic ornithine carbamoyltransferase activity in Pseudomonas species

\begin{tabular}{|c|c|c|c|c|c|}
\hline \multirow[b]{3}{*}{ Strain } & \multirow{3}{*}{$\begin{array}{l}\text { Oxygen } \\
\text { tension } \ldots\end{array}$} & \multicolumn{4}{|c|}{$\begin{array}{l}\text { Ornithine carbamoyltransferase } \\
\text { specific activity after growth on: }\end{array}$} \\
\hline & & \multicolumn{2}{|c|}{ Citrate $/ \mathrm{NH}_{4}^{+}$} & \multicolumn{2}{|c|}{ Arginine } \\
\hline & & High & Low & High & Low \\
\hline P. aeruginosa PAOI & & 15 & 800 & 12 & 860 \\
\hline P. putida IRC 204 & & 8 & 1990 & 25 & 1400 \\
\hline$P$. putida $\mathrm{P}_{2}$ ATCC 25571 & & 7 & 1310 & 18 & 730 \\
\hline P. putida ATCC 12633 & & 9 & 1440 & 20 & 740 \\
\hline P. fluorescens ATCC 13525 & & 7 & 390 & 6 & 125 \\
\hline P. mendocina NCIB 10541 & & 22 & 270 & 15 & 250 \\
\hline Aeromonas formicans NCIB 9232 & & 40 & 12 & 110 & 4300 \\
\hline Bacillus licheniformis IRC7 & & 25 & 15 & 1 & 1800 \\
\hline
\end{tabular}

Medium 154 was supplemented with $25 \mathrm{~mm}$-citrate and $10 \mathrm{~mm}-\left(\mathrm{NH}_{4}\right)_{2} \mathrm{SO}_{4}$, or $25 \mathrm{~mm}$-arginine as sole carbon and nitrogen source. High oxygen tension means air saturation in the fermenter, low oxygen tension means $<1 \%$ saturation (see Mercenier et al., 1980 b). The enzyme specific activity is expressed as $\mu$ mol citrulline formed $\mathrm{h}^{-1}$ (mg protein $)^{-1}$.

coli and $K$. aerogenes is characterized by urea excretion resulting from agmatinase activity (enzyme 8), whereas $P$. aeruginosa excretes carbamoylputrescine (Mercenier et al., 1980a). With the exception of $P$. cepacia, all Pseudomonas strains able to use agmatine as growth substrate excreted some $\mathrm{N}$-carbamoylputrescine produced by the action of an induced agmatine deiminase (enzyme 5) (Table 3). Arginine induced agmatine deiminase at low levels in most of the Pseudomonas strains, although the level was slightly higher in $P$. pseudoalcaligenes and was 10 -fold higher in $P$. mendocina and $P$. stutzeri. The in vivo tests revealed that all the strains exhibiting agmatine deiminase activity also possessed a $N$-carbamoylputrescine hydrolase (enzyme 6). Pseudomonas cepacia, E. coli, $K$. aerogenes and $B$. licheniformis gave no positive spots in this test (see Methods). Pseudomonas cepacia excreted urea when grown on agmatine as did $E$. coli and $K$. aerogenes. In contrast to the last two strains, no agmatinase activity was detected in crude extracts of $P$. cepacia after growth on agmatine; the only enzyme consistently detected was guanidinobutyrase (enzyme 13). The high level of this enzyme in cultures grown in the presence of agmatine indicated that agmatine acts as an inducer of guanidinobutyrase. The results suggest that these strains metabolize agmatine through a transaminase or an oxidase as reported for various fungi (Isobe et al., 1982). In addition, it should be noted that $P$. cepacia NCTC 10743 was able to use $N$-carbamoylputrescine as a carbon source and that $B$. licheniformis could utilize $N$ carbamoylputrescine as a nitrogen source. No putrescine carbamoyltransferase was detected in the strains listed in Table 1 . The pathway of $N$-carbamoylputrescine utilization in $P$. cepacia and $B$. licheniformis has yet to be investigated.

\section{The arginine deiminase pathway}

Previous work in our laboratory showed that the arginine deiminase pathway in $P$. aeruginosa PAO or Aeromonas formicans was induced by particular growth conditions such as a transition from high to low oxygen tension or depletion of carbon and phosphate sources (Mercenier et al., $1980 \mathrm{~b}$; Stalon et al., 1982). We detected the arginine deiminase pathway in all the fluorescent species of group I and in $P$. mendocina. In the presence of oxygen, the enzymes of the arginine deiminase pathway (enzymes 1, 2,3) remained at a low level of activity but were induced when the oxygen tension was lowered even in the absence of arginine in the growth medium (Table 4). It should be noted that the maximum specific activity of the catabolic ornithine carbamoyltransferase and the two other enzymes of the pathway, were generally lower for Pseudomonas cultures growing on arginine as sole carbon and nitrogen source than on citrate plus ammonia. Growth on arginine stopped prematurely due to an excessive increase in $\mathrm{pH}$ of the medium (between 8.2 and 8.5 ), in spite of the buffering capacity of the medium, caused by the excretion of ammonia and ornithine. Under low oxygen tension, all the strains possessing the arginine 
deiminase pathway converted $5-15 \%$ of the available arginine into citrulline and $85-90 \%$ into ornithine. When grown in the presence of arginine under high oxygen tension, they did not produce measurable levels of the two metabolites. In $A$. formicans and $B$. licheniformis however, both exogenous arginine and stress conditions were needed to induce the arginine deiminase pathway. Arginine deiminase and the inducible ornithine carbamoyltransferase were not demonstrated in the other Pseudomonas species listed in Table 1, even under conditions of energy depletion.

\section{CONCLUSIONS}

The present study has established that the genus Pseudomonas is heterogeneous with respect to the mechanism of arginine dissimilation and further, that heterogeneity exists within the individual subgroups.

The arginine decarboxylase/agmatine deiminase pathway is a characteristic of the Pseudomonas strains of group I as defined by Palleroni et al. (1974). This enzymic sequence fulfils the polyamine requirement when arginine is abundant. However, this pathway may also be a major route of arginine dissimilation in P. mendocina, $P$. stutzeri and $P$. pseudoalcaligenes since growth on arginine elicited the synthesis of agmatine deiminase in these strains.

The arginine oxidase pathway is probably the main arginine catabolic pathway during aerobic growth of $P$. putida strains, since exogenous arginine induced the synthesis of guanidinobutyrase and $90 \%$ of arginine consumed could be recovered as urea in the culture medium.

The arginine deiminase route was characteristic of the fluorescent group of Pseudomonas as well as $P$. mendocina. The arginine deiminase pathway in $B$. licheniformis (Broman et al., 1978), $P$. aeruginosa (Mercenier et al., 1980 b), Streptococcus faecalis (Simon et al., 1982) and Aeromonas formicans (Stalon et al., 1982) serves to generate ATP under energy depletion conditions. Pseudomonas putida possesses at least three pathways for arginine utilization. It seems likely that there may also exist three arginine catabolic pathways in P. aeruginosa, since mutants blocked in both the arginine decarboxylase and arginine deiminase pathways and also defective in guanidinobutyrase were able to use arginine as sole carbon and nitrogen source (D. Haas, personal communication). The nature of this unknown arginine catabolic pathway remains to be discovered. With the exception of 2-ketoarginine and agmatine utilization, none of the arginine catabolic pathways described in this study were detected in P. cepacia strains, and the route for the catabolism of arginine in $P$. cepacia remains to be determined.

We are grateful to Corinne Vander Wauven and Jean-Paul Simon for their help in some experiments. 2Ketoarginine was synthesized by J. P. Simon. We thank P. H. Clarke for reading and correcting the manuscript and D. Haas and T. Leisinger for helpful discussions. This work was supported by F.N.R.S. grant nr. E-137.

\section{REFERENCES}

Abdelal, A. T. H. (1979). Arginine catabolism by micro-organisms. Annual Retien of Microbiology 33, $139-168$.

ARChibaLD, R. M. (1944). Determination of citrulline and allantoin and demonstration of citrulline in blood plasma. Journal of Biological Chemistry 156, $121-142$.

Broman, K., Lauwers, N., Stalon, V. \& Wiame, J. M. (1978). Oxygen and nitrate utilization by Bacillus licheniformis of the arginase and arginine deiminase routes of arginine catabolism, and other factors affecting their syntheses. Journal of Bacteriology 135, 920-927.

Chov, C. C. \& Rodwell, V. (1972). Metabolism of basic amino acids in Pseudomonas putida: guanidobutyrate amido-hydrolase. Journal of Biological Chemistry 247, 4486-4490.
FrIEDRICH, B. \& MAGASANIK, B. (1978). Utilization of arginine by Klebsiella aerogenes. Journal of Bacteriology 133, 680-685.

Friedrich, B. \& MagaSaniK, B. (1979). Enzymes of agmatine degradation and control of their synthesis in Klebsiella aerogenes. Journal of Bacteriology 137, $1127-1133$.

IsOBE, K., TANI, Y. \& Yamada, H. (1982). A new enzyme, agmatine oxidase, from fungi. Agricultural and Biological Chemistry 46, 1345-1351.

MeISTER, A. (1952) Enzymatic preparation of aketo acids. Journal of Biological Chemistry 197, 309-317.

MeISTER, A. (1953). $\alpha$ Keto analogues of arginine, ornithine and lysine. Journal of Biological Chemistry 206, 577-585.

Mercenier, A., Simon, J. P., HaAs, D. \& Stalon, V. $(1980 a)$. Catabolism of L-arginine by Pseudomonas 
aeruginosa. Journal of General Microbiology 116, 381389.

Mercenier, A., Simon, J. P., Vander Wauven, C., HaAs, D. \& Stalon, V. $(1980 \mathrm{~b})$. Regulation of enzyme synthesis in the arginine deiminase pathway of Pseudomonas aeruginosa. Journal of Bacteriology 144, 159-163.

Miller, D. R. \& Rodwell, V. (1971) Metabolism of basic amino acids in Pseudomonas putida: intermediates in L-arginine catabolism. Journal of Biological Chemistry 246, 5053-5058.

Morris, D. R. \& PARdeE, A. B. (1971) Multiple pathways of putrescine biosynthesis in Escherichia coli. Journal of Biological Chemistry 241, 3129-3135.

Palleroni, N. J., Kunisawa, R., Contopoulou, R. \& Doudoroff, M. (1974). Nucleic acid homologies in the genus Pseudomonas. International Journal of Systematic Bacteriology 23, 333-339.

Rahman, M., Laverack, P. D. \& Clarke, P. H. (1980). The catabolism of arginine by Pseudomonas aeruginosa. Journal of General Microbiology 116, 371 380.

Simon, J. P., Wargnies, B. \& Stalon, V. (1982). Control of enzyme synthesis in the arginine deiminase pathway of Streptococcus faecalis. Journal of Bacteriology 150, 1085-1090.

Stalon, V., Ramos, F., Piérard, A. \& Wiame, J. M. (1967). The occurrence of a catabolic and an anabolic ornithine carbamoyltransferase in Pseudomonas fluorescens. Biochimica et biophysica acta 139 , 91-97.

Stalon, V., Ramos, F., Piérard, A. \& Wiame, J. M. (1972). Regulation of the catabolic ornithine carbamoyltransferase of Pseudomonas fluorescens: a comparison with the anabolic transferase and with a mutationally modified catabolic transferase. European Journal of Biochemistry 29, 25-35.

Stalon, V., Simon, J. P. \& Mercenier, A. (1982). Enzymes of arginine utilization and their formation in Aeromonas formicans NCIB 9232. Archives of Microbiology 133, 295-299.

Stanier, R. Y., Palleroni, N. J. \& Doudoroff, M. (1966). The aerobic Pseudomonas: a taxonomic study. Journal of General Microbiology 43, 159-271.

TABOR, H., TABOR, C. H. \& HAFNER, E. W. (1976). Convenient method for detecting ${ }^{14} \mathrm{CO}_{2}$ in multiple samples. Applications to rapid screening for mutants. Journal of Bacteriology 128, 485-486.

VANDERBilt, A. S., Gaby, N. S. \& Rodwell, V. (1975). Intermediates and enzymes between $\alpha$ ketoarginine and $\gamma$-guanidinobutyrate in the L-arginine catabolic pathway of Pseudomonas putida. Journal of Biological Chemistry 250, 5322-5329.

Weathers, P. S., Chee, H. L. \& Allen, M. M. (1978). Arginine catabolism in Aphanocapsa 6308. Archives of Microbiology 118, 1-6. 